\title{
Incontinencia Urinaria Femenina
}

\author{
II PARTE - MANEJO
}

\author{
Dr. Antonio Lomanto Morán*
}

\section{INTRODUCGION}

El mayor conocimiento de la Incontinencia Urinaria de Esfuerzo ha contribuido a una mejor selección de los casos tributarios de tratamiento quirúrgico, aunque todavía persisten algunas incógnitas. A pesar de haberse propuesto más de cincuenta procedimientos quirúrgicos para el tratamiento de la Incontinencia Urinaria de Esfuerzo, la frecuencia de recidivas varía entre el tres y veinte por ciento $(1,2,3,4,5,6,7)$. Como muchos otros autores, también consideramos que la mayoría de los resultados quirúrgicos requiere investigaciones pre-operatorias minuciosas que permitan la selección y la clasificación de los casos y la determinación de las relaciones patogénicas e hidromecánicas de la unión uretro-vesical.

Muchas de éstas operaciones fueron ideadas y propuestas en épocas en que no se disponía de la información actual acerca de la etiología de la incontinencia de esfuerzo. La idea básica se apoyaba en construir un ángulo uretro vesical posterior, cuando éste no existía y estrechar la uretra con el fin de impedir el escape de orina. Para unos las operaciones tenían por objeto liberar la uretra, alegando que se hallaba desviada por cicatrices, mientras que otros atribuian el éxito de cualquier operación a las cicatrices producidas por ella. La aplicación de cuerpos extraños por debajo de la uretra también ha sido uno de los recursos para el tratamiento de la incontinencia.

\footnotetext{
* Director Departamento de Ginecología y Obstetricia, Facultad de Medicina. Universidad Nàcional de Colombia. Jefe de Ginecología Clínica Fray Bartolomé de las Casas. Caja de Previsión Social de Bogotá.
}

Probablemente, el progreso en éste campo de la patología se deba a la mejoría de los procedimientos diagnósticos y a la selección correcta de los casos para ser tratados.

En éste capítulo nos dedicaremos al tratamiento de la Incontinencia Urinaria de Esfuerzo genuina, llamada también por otros autores verdadera.

\section{TERAPEUTICA NO QUIRURGICA DE LA INCONTINENCIA URINARIA}

\section{Ejercicios Perineales Isométricos}

En los últimos cincuenta años, por sugerencia del doctor Kegel, se han beneficiado muchas mujeres con los ejercicios perineales isométricos. Los músculos del diafragma pélvico, en especial los pubococcígeos, son los responsables de la calidad de los tejidos en ésta región y de la función satisfactoria de los esfínteres. Los ejercicios de cualquier grupo de músculos fomentan el tono del tejido y facilitan la función de las estructuras adyacentes. El soporte proporcionado por el diafragma pélvico es su característica principal. La flacidez de éstos músculos puede ocasionar consecuencias serias tanto para la circulación, como para la relación anatómica y función de otros órganos pélvicos. El entrenamiento del músculo pubococcígeo a pesar de ser un proceso sencillo, con mucha frecuencia alivia a las mujeres incontinentes.

Se recomienda que se efectúen cientos de contracciones cada día, repetidas en sesiones de quince a treinta cada media hora. Se tendría que mantener cada contracción por lo menos durante tres segundos, relajar, y luego intentar la próxima contrac- 
ción. Hay que advertir a la paciente que no hay necesidad de hacer fuerza, los controles se pueden realizar quincenalmente para valorar resultados.

\section{Estímulos Eléctricos}

Es indudable que la estimulación eléctrica del suelo pélvico en algunas circunstancias inhibe la incontinencia (Alexander, 1976; Harrison, 1976). No se ha dilucidado el mecanismo de acción aunque éstos autores sugieren con optimismo que los efectos son semejantes a los obtenidos con los procedimientos quirúrgicos. Sin embargo, los aditamentos electrónicos no han cumplido la promesa original y tienen papel comparativamente secundario en el tratamiento de la incontinencia.

\section{Aditamentos Mecánicos}

Se han presentado muchos aditamentos mecánicos ingeniosos para controlar la incontinencia. Los doctores Spring y Eduards, en 1975, idearon un artefacto que colocado intravaginal sostiene y eleva la unión uretro vesical. Tienen las desventajas de dificultades en la introducción y la retención, y como ventaja que pueden ser útiles cuando la cirugía ha fracasado o está contraindicada.

\section{Tratamiento Farmacológico}

Desde hace muchos años se sabe que los estrógenos tienen propiedades alpha adrenérgicas y por tal motivo son capaces de proporcionar el cierre uretral principalmente en el tercio proximal. Lógicamente esta medicación tiene una aplicación limitada y es indispensable conocer las concentraciones hormonales de la paciente. Se ha sugerido la utilización local durante quince días con descansos períodicos. No es recomendable la administración oral por los efectos colaterales que pueda ocasionar la medicación.

\section{Tratamiento Quirúrgico}

La comprensión de las bases del buen éxito en la cirugía para la incontinencia urinaria se debe a los estudios de Enhorning y colaboradores (8) quien comprobó que la continencia se logra cuando la unión uretro vesical y la porción proximal de la uretra se elevan hacia la zona intra-abdominal. La presión intra uretral en estado normal es mayor que la presión vesical y cualquier aumento de la presión abdominal se transmitirá igualmente a la vejiga y a la porción proximal de la uretra, lo cual conservará el gradiente de presión entre vejiga y uretra y asegurará presión positiva de cierre en cuello vesical y uretra.
Para valorar resultados en el tratamiento quirúrgico de la incontinencia debe tenerse en cuenta sólo los datos suministrados por la paciente, ya que por el temor de que no sea sometida a nueva intervención puede considerarse conforme con el procedimiento realizado. Se debe hacer una evaluación con 250 cc de orina y no con la vejiga llena, ojalá en posiciones de decúbito y de piés.

Los criterios para considerar curada una mujer, en lo que a tiempo se refiere, no debe ser nunca inferior a dos años y ojalá hasta cinco años.

A continuación describiremos los diferentes procedimientos quirúrgicos que han sido utilizados para la corrección de la incontinencia urinaria de esfuerzo.

\section{INTERVENCIONES VAGINALES}

1. En el año de 1988, Schultz, realiza la primera intervención vaginal para la corrección de la incontinencia, que consistió en la extirpación de la pared vaginal anterior. No existen datos detallados de éste procedimiento.

2. Donald en el mismo año efectuó con éxito la colporrafia anterior y posterior con amputación del cuello uterino. El procedimiento fue descrito por Fothergill y fue ratificado en 1933 por Fletcher Shauw quien sugirió que la intervención se bautizara con el nombre de la operación de Manchester.

3. En 1913, Howard Kelly, publicó la monografía Incontinencia de Orina en la Mujer y describió la lesión del tejido del cuello vesical desgarrado y relajado, suturándolos con dos o tres puntos de colchonero con seda delgada (9).

4. En 1949, Pacey, sugirió que cuando había prolapso se comprometía el músculo pubococcigeo y los ligamentos pubo cervicales y procedió a efectuar la corrección de los ligamentos cardinales, pubo cervicales y músculos pubo coccígeos (10).

5. En 1950, Ingelman-Sundberg, cortó el músculo pubo coccígeo pasando unas asas por debajo del cuello vesical y otras por la pared vaginal posterior. De los dos lados se disecaba el músculo bulbocavernoso y se suturaba en la línea media como apoyo de la porción distal de la uretra (11). 
Según el esquema de Green la incontinencia de tipo I, o sea aquella en la cual está comprometido el ángulo uretro vesical posterior debe ser corregida por vía vaginal. Tal vez es el único autor que da una efectividad de más del noventa por ciento de mejoría con la vía vaginal (12). El resto de los autores $(13,14$ y 15) no dan el sesenta por ciento de curación.

Hay acuerdo entre los autores que trabajan en incontinencia urinaria, que cuando existe ésta asociada con celes de la pared anterior de la vagina es preferible hacer la corrección de la incontinencia por vía alta y el cistocele por vía vaginal.

6. En 1976, Frewen, aplica el injerto obtenido de la aponeurosis del oblicuo mayor y se coloca debajo de la uretra con el fin de disminuir el calibre y brindar sostén a ésta y al cuello de la vejiga. (16) La cifra global de cura fue de noventa y uno por ciento con una vigilancia hasta de cinco años.

7. En 1975, Horn, Kover (17) describieron el implante de fibrina de buey en forma de habichuela, que se coloca transversalmente en la facia pubo cervical en la unión uretro vesical. Describen el 93\% de mejoría.

Podemos concluir los siguientes puntos sobre las características de los procedimientos vaginales en el tratamiento de la incontinencia urinaria de esfuerzo.

1. Consideramos que estarían indicados en aquellos casos en que existe descenso notable de las paredes vaginales; también cuando existen histeroceles.

2. Son procedimientos que teóricamente dan menos morbilidad con respecto a los métodos suprapúbicos.

3. Estadísticamente la efectividad es muy inferior en comparación con los procedimientos suprapúbicos en lo que se refiere a curación de la incontinencia a largo plazo.

\section{INTERVENCIONES SUPRAPUBICAS}

\section{Cabestrillos Uretrovesicales}

Inicialmente estos procedimientos se reservaron como recursos secundarios para pacientes en quienes la incontinencia no había curado por intervenciones vaginales. Sin embargo, ya se están ulizando como operación primaria, pues se sabe según las últimas investigaciones que a la mujer hay que darle en primer instancia el tratamiento quirúrgico que le ofrezca mayor efectividad.

Sin lugar a dudas son más complicados y suelen necesitar incisiones abdominales y vaginales y el objetivo que persigue es elevar la unión uretro-vesical. Los elementos que se usan son orgánicos y sintéticos.

1. En 1907, Giordano, (18) utilizó el músculo recto interno del muslo, al que separó de la inserción y conservando el nervio y el pedículo vascular lo hizo pasar por la aponeurosis de Colles alrededor de la uretra y se lo fijó al periostio del pubis del mismo lado.

2. Goebell (19), despegó los músculos piramidales de la cara anterior del pubis, pasó los cabos libres hacia abajo y los suturó debajo de la uretra en la unión uretro vesical. Posteriormente Frangenhelm, en 1914 y Stoeckel en 1921 utilizaron frondas aponeuróticas.

3. En 1942, Aldridge (20), utilizando bandas aponeuróticas del oblicuo mayor y pasándolas por los bordes internos de los músculos rectos abdominales las colocó por debajo de la uretra y del cuello vesical. Esta intervención tiene la particularidad de que cuando la paciente realiza un esfuerzo las frondas aponeuróticas se elevan y ascienden el cuello vesical. Como dificultad técnica se menciona el grado de elevación del cuello vesical. Se reportan del setenta y cinco al ochenta y cinco por ciento de mejoría.

4. En 1944, Studdiford (21), se valió de puntos de colchonero de catgut introducidos por los extremos que se unían debajo del cuello de la vejiga, de ésta manera se formó una repisa aponeurótica.

5. En 1947, Millin (22), sólo hizo incisión abdominal e introdujo la fronda aponeurótica a ciegas alrededor de la uretra por un túnel suburetral. Las bandas aponeuróticas están colocadas por los bordes laterales de los músculos rectos abdominales. El porcentaje de éxitos alcanza según el autor el ochenta y cinco por ciento. Como complicaciones lógicas se describieron perforaciones vesicales. 
6. En 1968, Moir (23) ideó una hamaca para sostener el cuello de la vejiga y uretra, valiéndose de una tira ancha de mersilene de dos y medio centímetros de ancho y trece centímetros de largo. Moir obtuvo una mejoría de $85 \%$.

\section{Suspensiones Uretro Vesicales}

A continuación describiremos una serie de intervenciones que se iniciaron en 1947 cuyo objetivo fundamental es la elevación del cuello vesical o tejido parauretrales a estructuras anteriores o anterolaterales de la cara posterior del pubis, de la índole de cartílago de las sínfisis púbica, periostio de la rama del pubis, vaina del recto o ligamento de Cooper.

Ventajas de éstas técnicas:

a. Los resultados a largo plazo para el tratamiento de la incontinencia son superiores a los realizados por vía vaginal.

b. Pueden realizarse simultáneamente con intervenciones para patología abdominal.

Desventajas:

a. Cuando se utiliza el periostio son frecuentes la osteítis y el dolor.

b. Posiblemente se necesitan intervenciones vaginales, cuando existen prolapsos de pared vaginal.

1. La primera intervención de suspensión uretro vesical fue publicada por Everard Williams (24) quien disecó el espacio retropúbico para movilizar la uretra y cuello de la vejiga colocando cuatro o más puntos a cada lado de la línea media entre la pared de la vejiga y la cara posterior del pubis. Aunque no existen estadísticas, los resultados no debieron ser satisfactorios, pues utilizó catgut simple y la técnica no cumple con los objetivos de ubicar el cuello intra-abdominal.

2. El procedimiento más utilizado hasta la fecha es el ideado por los autores Marshall, Marchetti y Krantz (25), quienes en 1949 publicaron el ochenta y nueve por ciento de éxito. La descripción original coloca tres puntos a cada lado de la uretra y el cuello de la vejiga; cada uno de los puntos incluye tejido parauretral, pared lateral de la uretra (no el interior) y se inserta dos veces (para obtener "mordida doble" en la pared vaginal). La sutura se saca después por el periostio de la rama superior del pubis o por pericondrio de la sínfisis púbica. Después de anudar los puntos pueden colocarse otros adicionales entre la superficie anterior de la vejiga y los músculos rectos, para brindar más elevación y sostén.

Las complicaciones principales fueron osteítis púbica y puntos que se cortan a través del periostio o pericondrio y se sueltan.

3. En 1961, Burch (26), con el fin de evitar las molestias que ocasiona la operación de Marshall, Marchetti, Krantz, utilizó la fascia paravaginal fijándola al ligamento de Cooper. Esta técnica es de las más utilizadas en la actualidad y tiene como ventaja sobre la anterior que no traumatiza el componente uretral. El autor reporta noventa y tres por ciento de éxito a diez años de seguimiento.

Con ésta técnica el autor informa la presencia de enterocele que alcanza aproximadamente el siete por ciento. Con base en éstos datos algunos complementan la intervención con la obliteración del fondo de saco de Douglas y aproximación de los ligamentos uterosacros (Moschcowitz).

4. En 1959, Pereyra (27), describió una operación para elevar la uretra con puntos dobles que permiten sujetar los tejidos parauretrales a la vaina del recto. En éste procedimiento se introduce una cánula angulada por detrás del pubis previa incisión abdominal al lado de la línea media, guiándola hasta las estructuras parauretrales para obtener la sutura y anudarla preaponeurótica. Con éste método es posible realizar simultáneamente procedimientos vaginales sin las incisiones abiertas suprapúbicas. El inconveniente mayor es el de ser un procedimiento ciego y de ahí que en las publicaciones informan lesiones vesicales. El autor reporta noventa y dos por ciento de éxito.

5. En el Servicio de Urología de la Universidad de California, el doctor Tanagho (28), sugirió que no era necesario desbridar todo el espacio de Retzius, pues era importante respetar el mecanismo esfinteriano periuretral y con éstos criterios puntualizó que solamente eran necesarios dos puntos parauretrales (distales) 
y dos puntos paracervicales (proximales). El doctor Tanagho no reporta estadísticas, pero en nuestro medio (29) con un seguimiento a cuatro años se reporta noventa y tres por ciento de éxito. El mayor inconveniente se plantea cuando existe cistocele pues solamente asciende el trayecto uretral.

6. En la Universidad de Texas, el doctor Powell (30), publica un procedimiento con el nombre de Uretrocistopexia Retropúbica por vía vaginal y consiste en emplear la técnica de Marshall, Marchetti, Krantz. Plantea como ventajas el tiempo quirúrgico, la posibilidad de realizar procedimientos vaginales simultáneos. La experiencia de los investigadores a nivel nacional reportan una incidencia de éxito del $94.7 \%$ con un seguimiento a cuatro años (31).

7. En 1980, el doctor Stamey (32) publica una serie de doscientos tres casos con un éxito del noventa y uno por ciento. Utiliza la técnica de Pereyra, según como se describió anteriormente, pero además de utilizar agujas para levantar las estructuras paracervicales, complementa la técnica con el empleo de procedimientos endoscópicos intraoperatorios, y en esta forma controlar en forma adecuada la ubicación del cuello vesical y a la vez comprobar la integridad vesical.

La experiencia de esta técnica en nuestro Servicio, con seguimiento a cuatro años, asciende al noventa y seis por ciento de éxitos (33).

\section{Objetivos de la Cirugía en la Incontinencia Urinaria}

1. Restablecer la posición de la porción proximal de la uretra y permitir que se transmita la presión intra-abdominal.

2. Aumentar la presión de cierre uretral.

3. Incrementar la longitud uretral funcional.

4. Reforzar la suficiencia del cuello vesical.

5. Aumentar el sostén del cuello vesical.

\section{CONCLUSIONES}

De acuerdo con las descripciones anteriormente expuestas, podemos concluir los siguientes pun- tos con relación al tratamiento de la incontinencia urinaria de esfuerzo en la mujer:

1. Los ejercicios perineales isométricos, ideados por Keegel, son de gran utilidad en aquellas mujeres con ésta patología en etapas incipientes.

2. El uso de estrógenos locales en aquellas mujeres en quienes por razones de orden cronológico, o de otra índole presentan concentraciones bajas hormonales de origen ovárico, son de una utilidad considerable.

3. Los aditamentos intravaginales ocupan un buen puesto en aquellos casos en los que no se pueden realizar procedimientos quirúrgicos como recurso para el tratamiento de la incontinencia urinaria.

4. Los estímulos eléctricos mencionados por varios autores, son sin lugar a dudas métodos que no tienen mayor aceptación, posiblemente por la vaga especificidad que tiene sobre las estructuras pélvicas.

5. Existen un buen número de fármacos que teniendo características alpha adrenérgicas, se pueden utilizar, pero no sólo los efectos colaterales sistémicos son un obstáculo para su empleo, sino por el hecho de que tienen que ser utilizados de por vida.

6. En lo concerniente a las técnicas quirúrgicas el objetivo fundamental de ellas se concentra en ubicar el cuello vesical y la uretra proximal en el sector intra-abdominal.

7. Un axioma en las sociedades que trabajan sobre el tema de la incontinencia, es que la primera operación que se le practica a una mujer que padece esta entidad nosológica, es aquella que ofrece estadísticamente la mayor posibilidad de éxito.

8. Los procedimientos quirúrgicos vaginales, los consideramos de valor para el tratamiento de la incontinencia, solamente en aquellos casos en los cuales no es posible emplear la vía alta. Posiblemente en un futuro, empleando la vía vaginal asociándola con las fijaciones retropúbicas, sea una alternativa para aquellos casos en los que existen prolapso e incontinencia urinaria. 
9. No somos partidarios de utilizar la vía vaginal inicialmente y si fracasa, apelar a la suprapúbica porque:

a. la segunda intervención es muy laboriosa y con alta incidencia de morbilidad.

b. estadísticamente es más efectiva la vía suprapúbica.

10. Las intervenciones vaginales no son ideales para la corrección de la incontinencia urinaria porque se están utilizando estructuras anatómicas que generalmente han sido dilaceradas por la multiparidad.

11. Siempre hemos sido partidarios de la vía suprapúbica por las siguientes razones:

\section{BIBLIOGRAFIA}

1. BETHOUX, A.; BORRY, S.: Les mechanisms statique viceraux pelviens chez femme a la lumiere de l'exploration fonctionelle du dispositif en position debout. Ann. Chir., 16, 887, 1962.

2. CAMPBELL, N.F.; HARRISON, J.H.: Urology. W.B. Saunders Co., Filadelfia, Londres, Toronto, 1970.

3. GREEN, T.H.; Jr.: Urinary stress incontinence: Diferential diagnosis, pathophysiology and management. Am. J. Obstet. Gynecol., 122, 368, 1975.

4. JEFFCOATE, T. N/A.: Effects of urethrocystopexy for stress incontinence. Surg. Gynecol. Obstet., 98, 743, 1954.

5. PEREYRA, A.J.; LEBHERZ, T.B.: Combined urethro-vesical suspension and vaginourethroplasty of stress incontinence. Obstet. Gynecol., 30, 537, 1967.

6. ROBERT, H.G.: Traite de technique chirurgical gynecologique. Masson Cle, París, 1969.

7. ULLERY, J.C.: Treatment of stress incontinence in female. Am. J. Obstet. Gynecol., 66, 85 3, 1953.

8. ENHORNING, G. (1961). Simultaneous recording of intravesical and intra-urethral pressure. Acta Chirurgiae Scandinavica, Supplement 276, 1-68.

9. KELLY, H.A. (1913). Incontinence of urine in Women. Urologic and Cutaneus Review 17, 291-293.

10. PACEY, K. (1949). Pathology and repair of genital prolapse. Journal of Obstetrics and Gynaecology of the British Empire, $56,1-15$.

11. INGELMAN-SUNDBERG, A. (1950). Plastic repair of the pelvic floor. Acta Gynaecologica Scandinavica, 30 (Supplement 7), 318-328.

12. GREEN, T.H. (1962). Development of a plan for the diagnosis and treatment of urinary stress incontinence. American Journal of Obstetrics and Gynecology, 83, 632-648.

13. HODGKINSON, C.P. (1970). Stress urinary incontinence. American Journal of Obstetrics and Gynecology, 108, 11411168.

14. LOW, J.A. (1967). The management of anatomic urinary incontinence by vaginal repair. American Journal of Obstetrics and Gynecology, 97, 308-315.

15. LOMAnTO, A. Cols. Operación de Kelly. Rev. Col. Obstet. Ginecol. Vol. XXXIV No. 1. Pág. 28, 1983.

16. FREWEN W.K. (1976). Urethral graft in stress incontinence. Communication to the Sixth Annual Congress - International Continence Society, Antwerp.

17. HORN, B.; KOVER, J. \& MARTON, I. (1975). Treatment of stress incontinence by a fibrin bioplast. British Journal of Obstetrics and Gynaecology, 82, 61-53. a. Estadísticamente es más efectiva que la vía vaginal.

b. Las estructuras que se utilizan para fijar los elementos parauretrales y paravesicales son resistentes, a diferencia de los que se utilizan en la vía vaginal, que como se dijo son flácidos.

c. Si evaluamos la ubicación de la uretra proximal y el cuello vesical, podemos decir que sí se obtiene su ubicación intra-abdominal.

d. Cuando se utiliza suturas del tipo no absorbible se ha comprobado una eficacia mayor a largo plazo.

18. GIORDANO, D. (1970). 20th Congress Francais de Chirugie. Quoted by Ullery, J.C. (1953). Stress Incontinence in the female. New York: Grune and Stratton.

19. GOEBELL, R. (1910). Zur operative Beseitigung der angeborenen incontinentia Vesciae. Zeitschrift für gynäkologische Urologie, 2, 187.

20. ALDRIDGE, A.H. (1942). Transplantation of fascia for relief of urinary stress incontinence. American Journal of Obstetrics and Gynecology, 44, 398-411.

21. STUDDIFORD (1944). Transplantation of abdominal fascia for relief of urinary stress incontinence. American Journal of Obstetrics and Gynecology, 47. 764-774.

22. MILLIN, T. (1947). Discussion on stress incontinence in micturition. Proceedings of the Royal Society of Medicine, 40, 361-370.

23. MOIR, J.C. (1947). Discussion on stress incontinence in micturition. Proceedings of the Royal Society of Medicine, 40, 367-369.

24. WILLIAMS, E. (1947). Discussion on stress incontinence. Proceedings of the Royal Society of Medicine, 40, 361-370.

25. MARSHALL, V.F.; MARCHETTI, A.A.; \& KRANTZ, K.E. (1949). The correction of stress incontinence by simple vesico-urethral suspension Surgery. Gynecology and Obstetrics, 88, 509-518.

26. BURCH, J.C. (1961). Urethro-vaginal fixation to Cooper's ligament for correction of stress incontinence, cystocele and prolapse. American Journal of Obstetrics and Gynecology, 81, 281-290.

27. PEREYRA, A.J.; \& LEBHERZ, T.B. (1967). Combined urethro-vesical suspension and vagino-urethroplasty for correction of urinary stress incontinence. Obstetrics and Gynecology, 30 537-546.

28. TANAGHO, E.A.: Colpocistourethropexy. The way we do it. J. Urology, 110: 751-3, Dec. 1976.

29. LOMANTO, A. y Cols. Evaluación de la Colpocistouretropexia en la Incontinencia Urinaria de Esfuerzo. Rev. Col. Obstet. y Ginecol. Vol. XXXIII, Pág. 112, 1962.

30. POWELL, L.C. Jr. Retropubic Urethrocystopexy: Vaginal approach. Am. J. Obstet. Gynecol. 140: 91, 1981.

31. REDONDO H. y Cols. Uretrocistopexia Infrapúbica. Rev. Col. Obstet. y Ginecol. Vol. XXXIX, No. 1. pág. 43, enero-marzo, 1988.

32. STAMEY, T.A. Surgery of Female Incontinence. Pág. 77, 1980.

33. LOMANTo, A. y Cols. La Cervicosuspensión Endoscópica en el Tratamiento de la Incontinencia Urinaria de Esfuerzo. Rev. Col. Obstet. y Ginecol. Vol. XXXVIII, No. 5. pág. 331, Septiembre-Octubre 1987. 